\title{
EXISTENCE OF MULTIPLE SOLUTIONS OF SOME SECOND ORDER IMPULSIVE DIFFERENTIAL EQUATIONS
}

\author{
Jing XiaO — Juan J. Nieto — Zhiguo Luo
}

ABSTRACT. This paper uses critical point theory and variational methods to investigate the multiple solutions of a boundary value problem for second order impulsive differential equations. The conditions for the existence of multiple solutions are established.

\section{Introduction}

Consider the following nonlinear boundary value problems for second order impulsive differential equation:

$$
\begin{cases}\left(p(t) u^{\prime}(t)\right)^{\prime}+q(t) u(t)+f(t, u(t))=0, & \text { a.e. } t \in J^{\prime}, \\ -\Delta u^{\prime}\left(t_{j}\right)=I_{j}\left(u\left(t_{j}\right)\right), & j=1, \ldots, n, \\ u(0)=0, \quad a_{1} u(1)+u^{\prime}(1)=0, & \end{cases}
$$

where $J=[0,1], 0=t_{0}<t_{1}<\ldots<t_{n}<t_{n+1}=1, J^{\prime}=J \backslash\left\{t_{1}, \ldots, t_{n}\right\}$, $f \in C[J \times \mathbb{R}, \mathbb{R}], p(t) \in C^{1}[0,1], q(t) \in C[0,1]$, with $0<m \leq p(t) \leq M$, $q(t)+p(t) \leq 0$ on $J$, the constant $a_{1}>-m / p(1), \Delta u^{\prime}\left(t_{j}\right)=u^{\prime}\left(t_{j}^{+}\right)-u^{\prime}\left(t_{j}^{-}\right)$for

2010 Mathematics Subject Classification. 34B15, 34B18, 34B37, 58E30.

Key words and phrases. Boundary value problem with impulses, critical point theory, variational methods.

This work is supported by the National Nature Science Foundation P.R. China (10871063), and Nature Science Foundation of Guangdong Medical College(B2012053), partially supported by Ministerio de Educacion y Ciencia and FEDER, Project MTM2007- 61724, and MTM201015314, and by Xunta de Galicia and FEDER, Project PGIDIT06PXIB207023PR. 
$u^{\prime}\left(t_{j}^{-}\right)$and $u^{\prime}\left(t_{j}^{+}\right)$, respectively, the left and right limits of $u^{\prime}(t)$ at $t_{j}, j=1, \ldots, n$, $1 \leq n<+\infty$.

The theory of impulsive differential equations has been emerging as an important area of investigation in recent years. Some classical tools such as coincidence degree theory of Mawhin, fixed point theorems in cones, and the method of lower and upper solutions have been used widely to get positive solutions of impulsive differential equations. For the theory and classical results see the monographs [1], [3], [12], [17]. Some recent development and applications of impulsive differential equations can been seen in [2], [5], [7], [9], [14], [16], [19], [20]. We point out that in a second order differential equation $u^{\prime \prime}=f\left(t, u, u^{\prime}\right)$, one usually considers impulses in the position $u$ and the velocity $u^{\prime}$. However, in the motion of spacecraft one has to consider instantaneous impulses depending on the position that result in jump discontinuities in velocity, but with no change in position [4]. The impulses only on the velocity occurs also in impulsive mechanics [10].

Recently, taking a Dirichlet problem with impulses as a model, Nieto and O'Regan [8] have shown that the impulsive problem minimize some (energy) functional, and the critical points of that functional are indeed solutions of the impulsive problem. Inspired by the work [8], in this paper we use critical point theory and variational methods to investigate the multiple solutions of (1.1). our main results extend the study made in [8], [13], [15], [21] in the sense that we deal with a class of problems that is not considered in those papers.

The rest of the paper is organized as follows: In Section 2, we give several important lemmas. The main theorems are formulated and proved in Section 3. And in Section 4, we give an example to demonstrate the application of our results.

\section{Preliminaries}

We now state some celebrated results in nonlinear functional analysis and critical point theory. Suppose that $X$ is a Banach space (in particular a Hilbert space) and $\varphi: X \rightarrow \mathbb{R}$ is differentiable. We say that $\varphi$ satisfies the Palais-Smale condition if every sequence $\left\{u_{k}\right\}$ in the space $X$ such that $\left\{\varphi\left(u_{k}\right)\right\}$ is bounded and $\lim _{k \rightarrow \infty} \varphi^{\prime}\left(u_{k}\right)=0$ contains a convergent subsequence.

Lemma 2.1 (Mountain Pass Theorem [6, Theorem 4.10]). Let $\varphi \in C^{1}(X, \mathbb{R})$. Assume that there exist $u_{0}, u_{1} \in X$ and a bounded neighbourhood $\Omega$ of $u_{0}$ such that $u_{1}$ is not in $\Omega$ and

$$
\inf _{v \in \partial \Omega} \varphi(v)>\max \left\{\varphi\left(u_{0}\right), \varphi\left(u_{1}\right)\right\} .
$$

Then there exists a critical point $u$ of $\varphi$ i.e. $\varphi^{\prime}(u)=0$, with

$$
\varphi(u)>\max \left\{\varphi\left(u_{0}\right), \varphi\left(u_{1}\right)\right\}
$$


Note that if either $u_{0}$ or $u_{1}$ is a critical point of $\varphi$ then we obtain the existence of at least two critical points for $\varphi$.

Lemma 2.2 ([19, Theorem 38.A]). For the functional $F: M \subseteq X \rightarrow R$ with $M$ not empty, $\min _{u \in M} F(u)=a$ has a solution in case the following hold:

(a) $X$ is a real reflexive Banach space,

(b) $M$ is bounded and weak sequentially closed,

(c) $F$ is weak sequentially lower semi-continuous on $M$, i.e. by definition, for each sequence $\left\{u_{k}\right\}$ in $M$ such that $u_{k} \rightarrow u$ as $k \rightarrow \infty$, we have $F(u) \leq \varliminf_{k \rightarrow \infty} F\left(u_{k}\right)$ holds.

Lemma 2.3 ([11, Theorem 9.12]). Let $E$ be an infinite dimensional real Banach space and $\varphi \in C^{1}(E, \mathbb{R})$ be even, satisfying the Palais-Smale condition and $\varphi(0)=0$. If $E=V \oplus X$, where $V$ is finite dimensional, and $\varphi$ satisfies the following conditions:

(a) There exist constants $\rho, \sigma>0$ such that $\left.\varphi\right|_{\partial B_{\rho} \cap X} \geq \sigma$,

(b) For each finite dimensional subspace $V_{1} \subset E$, there is an $R=R\left(V_{1}\right)$ such that $\varphi(u) \leq 0$ for every $u \in V_{1}$ with $\|u\|>R$.

Then $\varphi$ has an unbounded sequence of critical values.

Consider the Hilbert spaces $H=\left\{u \in H^{1}(0,1): u(0)=0\right\}$ with the inner product and norm

$$
\begin{aligned}
(u, v) & =\int_{0}^{1} p(t)\left(u^{\prime}(t) v^{\prime}(t)+u(t) v(t)\right) d t \\
\|u\| & =\left(\int_{0}^{1} p(t)\left(\left|u^{\prime}(t)\right|^{2}+|u(t)|^{2}\right) d t\right)^{1 / 2} .
\end{aligned}
$$

Lemma 2.4. If $u \in H$, then

$$
\|u\|_{0} \leq \frac{1}{\sqrt{m}}\|u\|, \quad \text { where }\|u\|_{0}=\max _{t \in[0,1]}|u(t)| .
$$

PROOF. The result is followed by the following inequalities:

$$
|u(t)| \leq \int_{0}^{1}\left|u^{\prime}(t)\right| d t \leq\left(\int_{0}^{1} \frac{1}{p(t)} d t\right)^{1 / 2}\left(\int_{0}^{1} p(t)\left|u^{\prime}(t)\right|^{2} d t\right)^{1 / 2} \leq \frac{1}{\sqrt{m}}\|u\| .
$$

For $u \in H$, we have that $u$ is absolutely continuous, and $u^{\prime} \in L^{2}(0,1)$. In this case, the one-sided derivatives $u^{\prime}\left(t^{-}\right), u^{\prime}\left(t^{+}\right)$may not exist. If $u \in H^{2}(0,1)$, then $u$ and $u^{\prime}$ are absolutely continuous, and $u^{\prime \prime} \in L^{2}(0,1)$. Hence $\Delta u^{\prime}(t)=$ $u^{\prime}\left(t^{+}\right)-u^{\prime}\left(t^{-}\right)=0$ for any $t \in J$. So by a classical solution of (1.1) we mean a function $u \in C(0,1)$ satisfying the following conditions:

- For every $j=0,1, \ldots, n, u_{j}=u_{\mid\left(t_{j}, t_{j+1}\right)} \in H^{2}\left(t_{j}, t_{j+1}\right)$; 
- $u$ satisfies the boundary condition of (1.1);

- $u^{\prime}\left(t_{j}^{-}\right), u^{\prime}\left(t_{j}^{+}\right), j=1,2, \ldots, n$, exist and the impulsive conditions of (1.1) hold;

- $u$ satisfies the first equation of (1.1) for $t \in J^{\prime}$.

Take $v \in H$ and multiply the first equation of (1.1) by $v$ and integrate it between 0 and 1 :

$$
\int_{0}^{1}\left(p(t) u^{\prime}\right)^{\prime} v d t+\int_{0}^{1} q(t) u v d t+\int_{0}^{1} f(t, u) v d t=0 .
$$

This leads to

$$
\begin{aligned}
\int_{0}^{1}\left[p(t)\left(u^{\prime} v^{\prime}+u v\right)-(q(t)\right. & +p(t)) u v-f(t, u) v] d t \\
& =\sum_{j=1}^{n} p\left(t_{j}\right) v\left(t_{j}\right) I_{j}\left(u\left(t_{j}\right)\right)-a_{1} p(1) u(1) v(1) .
\end{aligned}
$$

Thus, a weak solution for the impulsive problem (1.1) is a function $u \in H$ such that (2.1) holds for any $v \in H$.

Now, define $G(t, u)=\int_{0}^{u} f(t, \xi) d \xi$. We consider the functional $\varphi: H \rightarrow \mathbb{R}$, defined by

$$
\begin{aligned}
\varphi(u)=\int_{0}^{1}\left[\frac{1}{2} p(t)\left(\left|u^{\prime}\right|^{2}+|u|^{2}\right)\right. & \left.-\frac{1}{2}(q(t)+p(t)) u^{2}-G(t, u)\right] d t \\
& -\sum_{j=1}^{n} p\left(t_{j}\right) \int_{0}^{u\left(t_{j}\right)} I_{j}(t) d t+\frac{1}{2} a_{1} p(1) u^{2}(1) .
\end{aligned}
$$

It is clear that $\varphi$ is differentiable at any $u \in H$ and

$$
\begin{aligned}
\varphi^{\prime}(u)(v)=\int_{0}^{1}\left[p(t)\left(u^{\prime} v^{\prime}+u v\right)\right. & -(q(t)+p(t)) u v-f(t, u) v] d t \\
& -\sum_{j=1}^{n} p\left(t_{j}\right) v\left(t_{j}\right) I_{j}\left(u\left(t_{j}\right)\right)+a_{1} p(1) u(1) v(1) .
\end{aligned}
$$

Hence, a critical point of $\varphi$, defined by (2.2), gives us a weak solution of (1.1).

Lemma 2.5. If $u \in H$ is a weak solution of (1.1), then $u$ is a classical solution of (1.1).

Proof. The proof is similar to [3]. For integrity, we state it as follows. If $u \in H$ is a weak solution of (1.1), then by the definition of weak solution, equation (2.1) holds for all $v \in H$. For $j \in\{1, \ldots, n\}$, choose $v \in H$ with $v(t)=0$ for every $t \in\left[0, t_{j}\right] \cup\left[t_{j+1}, 1\right]$. Then

$$
\int_{t_{j}}^{t_{j+1}}\left[p(t)\left(u^{\prime} v^{\prime}+u v\right)-(q(t)+p(t)) u v-f(t, u) v\right] d t=0 .
$$


By the definition of weak derivative, the above equality implies

$$
\left(p(t) u^{\prime}(t)\right)^{\prime}+q(t) u+f(t, u)=0 \quad \text { for a.e. } t \in\left(t_{j}, t_{j+1}\right) .
$$

Hence $u_{j} \in H^{2}\left(t_{j}, t_{j+1}\right)$ and $u$ satisfies the first equation of (1.1) almost everywhere on $[0,1]$. Now multiplying by $v \in H$ and integrating between 0 and 1 , we get

$$
-\sum_{j=1}^{n} \Delta u^{\prime}\left(t_{j}\right) v\left(t_{j}\right)=\sum_{j=1}^{n} I_{j}\left(u\left(t_{j}\right)\right) v\left(t_{j}\right) .
$$

Hence, $-\Delta u^{\prime}\left(t_{j}\right)=I_{j}\left(u\left(t_{j}\right)\right)$, for every $j=1, \ldots, n$. So $u$ satisfies the impulsive conditions of (1.1). Similarly, $u$ satisfies the boundary condition. Therefore, $u$ is a solution of problem (1.1).

Let

$$
a(u, v)=\int_{0}^{1}\left[p(t)\left(u^{\prime} v^{\prime}+u v\right)-(q(t)+p(t)) u v\right] d t+a_{1} p(1) u(1) v(1)
$$

Then we have

LEMMA 2.6. If $a_{1}>-m / p(1)$, there exist constants $\beta>\alpha>0$ such that

$$
\alpha\|u\|^{2} \leq a(u, u) \leq \beta\|u\|^{2}, \quad u \in H .
$$

ProOF. Firstly we proof the left part of (2.4):

$$
\begin{aligned}
a(u, u) & =\int_{0}^{1}\left[p(t)\left(\left|u^{\prime}\right|^{2}+|u|^{2}\right)-(q(t)+p(t)) u^{2}\right] d t+a_{1} p(1) u^{2}(1) \\
& \geq\|u\|^{2}+a_{1} p(1) u^{2}(1) .
\end{aligned}
$$

If $a_{1} \geq 0$, then we have $a(u, u) \geq\|u\|^{2}$. And if $0>a_{1}>-m / p(1)$, then by Lemma 2.4 we have $a(u, u) \geq\left(1+a_{1} p(1) / m\right)\|u\|^{2}$.

On the other hand, since $p(t), q(t)$ are continuous in $[0,1]$, then there exists a constant $c_{1}>0$ such that $p(t)+q(t)>-c_{1} p(t)$. So, by Lemma 2.4 , we have

$$
\begin{aligned}
a(u, u) & =\int_{0}^{1}\left[p(t)\left(\left|u^{\prime}\right|^{2}+|u|^{2}\right)-(q(t)+p(t)) u^{2}\right] d t+a_{1} p(1) u^{2}(1) \\
& \leq\|u\|^{2}+c_{1}\|u\|^{2}+a_{1} p(1) u^{2}(1) \leq\left(1+c_{1}+\left|a_{1}\right| \frac{M}{m}\right)\|u\|^{2}
\end{aligned}
$$

Lemma 2.7. The function $\varphi: H \rightarrow \mathbb{R}$ defined by (2.2) is continuous, continuously differentiable and weakly lower semi-continuous. Moreover, it satisfies the Palais-Smale condition, if the following condition holds:

$\left(\mathrm{H}_{1}\right)$ For all $u \in H, \mu G(t, u) \leq u f(t, u)$, and $I_{j}(u) u \geq \mu \int_{0}^{u} I_{j}(s) d s$, where $j=1, \ldots, n, \mu>2 \beta / \alpha(\alpha, \beta$ are defined in Lemma 2.6). 
Proof. Using the continuity of $f$ and $I_{j}, j=1, \ldots, n$, we obtain the continuity and differentiability of $\varphi$ and $\varphi^{\prime}$. To show that $\varphi$ is weakly lower semicontinuous, let $u_{k}$ weakly convergent to $u$ in $H$. Then $\|u\| \leq \underset{k \rightarrow \infty}{\varliminf_{k}}\left\|u_{k}\right\|$, and $\left\{u_{k}\right\}$ converges uniformly to $u$ in $C[0,1]$. So when $k \rightarrow \infty$, we have

$$
\begin{aligned}
& \int_{0}^{1}\left[-\frac{1}{2}(q(t)+p(t)) u_{k}^{2}-G\left(t, u_{k}\right)\right] d t-\sum_{j=1}^{n} p\left(t_{j}\right) \int_{0}^{u_{k}\left(t_{j}\right)} I_{j}(t) d t+\frac{1}{2} a_{1} p(1) u_{k}^{2}(1) \\
& \rightarrow \int_{0}^{1}\left[-\frac{1}{2}(q(t)+p(t)) u^{2}-G(t, u)\right] d t-\sum_{j=1}^{n} p\left(t_{j}\right) \int_{0}^{u\left(t_{j}\right)} I_{j}(t) d t+\frac{1}{2} a_{1} p(1) u^{2}(1) .
\end{aligned}
$$

We conclude that $\varphi(u) \leq \varliminf_{k \rightarrow \infty} \varphi\left(u_{k}\right)$. Then $\varphi$ is weakly lower semi-continuous.

To show that $\varphi$ satisfies the Palais-Smale condition, let $\left\{\varphi\left(u_{k}\right)\right\}$ be a bounded sequence such that $\lim _{k \rightarrow \infty} \varphi^{\prime}\left(u_{k}\right)=0$. Now we show that $\left\|u_{k}\right\|$ is bounded. By (2.3) we have

$$
\begin{aligned}
\int_{0}^{1} f\left(t, u_{k}\right) u_{k} d t & =\int_{0}^{1}\left[p(t)\left(\left|u_{k}^{\prime}\right|^{2}+\left|u_{k}\right|^{2}\right)-(q(t)+p(t)) u_{k}^{2}\right] d t \\
& -\varphi^{\prime}\left(u_{k}\right)\left(u_{k}\right)-\sum_{j=1}^{n} p\left(t_{j}\right) u_{k}\left(t_{j}\right) I_{j}\left(u_{k}\left(t_{j}\right)\right)+a_{1} p(1) u_{k}^{2}(1) .
\end{aligned}
$$

From (2.4), (2.5) and condition $\left(\mathrm{H}_{1}\right)$, we get

$$
\begin{aligned}
\varphi\left(u_{k}\right) & \geq \frac{\alpha}{2}\left\|u_{k}\right\|^{2}-\int_{0}^{1} G\left(t, u_{k}\right) d t-\sum_{j=1}^{n} p\left(t_{j}\right) \int_{0}^{u_{k}\left(t_{j}\right)} I_{j}(t) d t \\
& \geq \frac{\alpha}{2}\left\|u_{k}\right\|^{2}-\frac{1}{\mu} \int_{0}^{1} u_{k} f\left(t, u_{k}\right) d t-\sum_{j=1}^{n} p\left(t_{j}\right) \int_{0}^{u_{k}\left(t_{j}\right)} I_{j}(t) d t \\
& \geq\left(\frac{\alpha}{2}-\frac{\beta}{\mu}\right)\left\|u_{k}\right\|^{2}+\frac{1}{\mu} \varphi^{\prime}\left(u_{k}\right)\left(u_{k}\right) \\
& \geq\left(\frac{\alpha}{2}-\frac{\beta}{\mu}\right)\left\|u_{k}\right\|^{2}-\frac{1}{\mu}\left\|\varphi^{\prime}\left(u_{k}\right)\right\|\left\|\left(u_{k}\right)\right\| .
\end{aligned}
$$

Since $\varphi\left(u_{k}\right)$ is bounded, by (2.6) we have $\left\|u_{k}\right\|$ is bounded.

Since $H$ is a reflexive Banach space, there exists a subsequence of $\left\{u_{k}\right\}$ (for simplicity denoted again by $\left\{u_{k}\right\}$ ) such that $\left\{u_{k}\right\}$ weakly converges to some $u$ in $H$. Then the sequence $\left\{u_{k}\right\}$ converges uniformly to $u$ in $C[0,1]$.

By (2.5), we have

$$
\begin{aligned}
\left\|u_{k}\right\|^{2}=\int_{0}^{1}\left[(q(t)+p(t)) u_{k}^{2}+\right. & \left.f\left(t, u_{k}\right) u_{k}\right] d t+\varphi^{\prime}\left(u_{k}\right)\left(u_{k}\right) \\
& +\sum_{j=1}^{n} p\left(t_{j}\right) u_{k}\left(t_{j}\right) I_{j}\left(u_{k}\left(t_{j}\right)\right)-a_{1} p(1) u_{k}^{2}(1) .
\end{aligned}
$$


So when $k \rightarrow \infty$, we have

$\left\|u_{k}\right\|^{2} \rightarrow \int_{0}^{1}\left[(q(t)+p(t)) u^{2}+f(t, u) u\right] d t+\sum_{j=1}^{n} p\left(t_{j}\right) u\left(t_{j}\right) I_{j}\left(u\left(t_{j}\right)\right)-a_{1} p(1) u^{2}(1)$.

Then $\left\|u_{k}\right\|$ converges in $H$.

Since $H$ is a Hilbert space, and the sequence $\left\{u_{k}\right\} \in H$ satisfies $u_{k} \rightarrow u$, then $\left\{u_{k}\right\}$ converges (strongly) to $u$, i.e. $u_{k} \rightarrow u$. $\varphi$ satisfies the Palais-Smale condition.

\section{Main results}

For convenience and simplicity in the following discussions, we use the notations:

$$
\begin{array}{rlrl}
G^{0}(t, u)=\limsup _{u \rightarrow 0} \frac{G(t, u)}{|u|^{\mu}}, & & I^{0}(j)=\limsup _{u \rightarrow 0} \frac{1}{|u|^{\mu}} \int_{0}^{u} I_{j}(t) d t, \\
G_{\infty}(t, u)=\liminf _{u \rightarrow \infty} \frac{G(t, u)}{|u|^{\mu}}, & I_{\infty}(j)=\liminf _{u \rightarrow \infty} \frac{1}{|u|^{\mu}} \int_{0}^{u} I_{j}(t) d t .
\end{array}
$$

Theorem 3.1. Suppose $\left(\mathrm{H}_{1}\right)$ and $a_{1}>-m / p(1)$ hold. Then the BVP (1.1) has at least two positive solutions if the following condition holds:

$\left(\mathrm{H}_{2}\right)$ There exists $\delta, \delta_{j}, \gamma, \gamma_{j}>0$ such that $G^{0}(t, u) \leq \delta, I^{0}(j) \leq \delta_{j}$ and $G_{\infty}(t, u) \geq \gamma, I_{\infty}(j) \geq \gamma_{j}$, where $j=1, \ldots, n$.

Proof. Firstly, we apply Lemma 2.2 to show that there exists $T$ such that the functional $\varphi$ has a local minimum $u_{0} \in B_{T}=\{u \in H:\|u\|<T\}$. For every $T>0$, since $H$ is a Hilbert space, it is easy to deduce that $\bar{B}_{T}$ is a bounded and weak sequentially closed. Lemma 2.7 has shown that $\varphi$ is weak sequentially lower semi-continuous on $\bar{B}_{T}$. Besides, $H$ is a reflexive Banach space, so by Lemma 2.2 we can have this $u_{0}$ such that $\varphi\left(u_{0}\right)=\min \left\{\varphi(u): u \in \bar{B}_{T}\right\}$.

Now we will show that $\varphi\left(u_{0}\right)<\inf \left\{\varphi(u): u \in \partial B_{T}\right\}$, for some $T=T_{0}$. In fact, by $\left(\mathrm{H}_{2}\right)$ we can choose $T_{0}, \varepsilon>0$ satisfying

$$
\begin{gathered}
\int_{0}^{u} I_{j}(t) d t \leq \delta_{j}|u|^{\mu}, \quad G(t, u) \leq \delta|u|^{\mu}, \quad \text { for }\|u\| \leq T_{0}, \\
\frac{\alpha}{2} T_{0}^{2}-\left(\delta+\sum_{j=1}^{n} \delta_{j} p\left(t_{j}\right)\right)\left(\frac{T_{0}}{\sqrt{m}}\right)^{\mu}>\varepsilon .
\end{gathered}
$$

For any $u \in \partial B_{T_{0}},\|u\|=T_{0}$, we have

$$
\begin{aligned}
\varphi(u)= & \int_{0}^{1}\left[\frac{1}{2} p(t)\left(\left|u^{\prime}\right|^{2}+|u|^{2}\right)-\frac{1}{2}(q(t)+p(t)) u^{2}-G(t, u)\right] d t \\
& -\sum_{j=1}^{n} p\left(t_{j}\right) \int_{0}^{u\left(t_{j}\right)} I_{j}(t) d t+\frac{1}{2} a_{1} p(1) u^{2}(1)
\end{aligned}
$$




$$
\begin{aligned}
& \geq \frac{\alpha}{2}\|u\|^{2}-\int_{0}^{1} G(t, u) d t-\sum_{j=1}^{n} p\left(t_{j}\right) \int_{0}^{u\left(t_{j}\right)} I_{j}(t) d t \\
& \geq \frac{\alpha}{2}\|u\|^{2}-\delta \int_{0}^{1}|u|^{\mu} d t-\sum_{j=1}^{n} \delta_{j} p\left(t_{j}\right)\left|u\left(t_{j}\right)\right|^{\mu} \\
& \geq \frac{\alpha}{2}\|u\|^{2}-\frac{\delta\|u\|^{\mu}}{(\sqrt{m})^{\mu}}-\sum_{j=1}^{n} \frac{\delta_{j} p\left(t_{j}\right)\|u\|^{\mu}}{(\sqrt{m})^{\mu}} \\
& =\frac{\alpha}{2} T_{0}^{2}-\left(\delta+\sum_{j=1}^{n} \delta_{j} p\left(t_{j}\right)\right)\left(\frac{T_{0}}{\sqrt{m}}\right)^{\mu}>\varepsilon .
\end{aligned}
$$

So $\varphi(u)>\varepsilon$ for any $u \in \partial B_{T_{0}}$. Besides, $\varphi\left(u_{0}\right) \leq \varphi(0)=0$. Then $\varphi(u)>\varepsilon>$ $\varphi(0) \geq \varphi\left(u_{0}\right)$ for any $u \in \partial B_{T_{0}}$. So $\varphi\left(u_{0}\right)<\inf \left\{\varphi(u): u \in \partial B_{T_{0}}\right\}$. Hence, $\varphi$ has a local minimum $u_{0} \in B_{T_{0}}=\left\{u \in H:\|u\|<T_{0}\right\}$.

Next, we will show that there exists $u_{1}$ with $\left\|u_{1}\right\|>T_{0}$ such that $\varphi\left(u_{1}\right)<$ $\inf \left\{\varphi(u): u \in \partial B_{T_{0}}\right\}$.

For the above $T_{0}$, by $\left(H_{2}\right)$, we can choose a sufficiently large $T_{1}$, such that For all $\|u\| \geq T_{1}>T_{0}$ satisfying $G(t, u) \geq \gamma|u|^{\mu}$ and $\int_{0}^{u} I_{j}(t) d t \geq \gamma_{j}|u|^{\mu}$. Then for all $\|u\| \geq T_{1}>T_{0}$ we have

$$
\begin{aligned}
\varphi(u)= & \int_{0}^{1}\left[\frac{1}{2} p(t)\left(\left|u^{\prime}\right|^{2}+|u|^{2}\right)-\frac{1}{2}(q(t)+p(t)) u^{2}-G(t, u)\right] d t \\
& -\sum_{j=1}^{n} p\left(t_{j}\right) \int_{0}^{u\left(t_{j}\right)} I_{j}(t) d t+\frac{1}{2} a_{1} p(1) u^{2}(1) \\
\leq & \frac{\beta}{2}\|u\|^{2}-\int_{0}^{1} G(t, u) d t-\sum_{j=1}^{n} \gamma_{j} p\left(t_{j}\right)\left|u\left(t_{j}\right)\right|^{\mu} \\
\leq & \frac{\beta}{2}\|u\|^{2}-\gamma \int_{0}^{1}|u|^{\mu} d t-\sum_{j=1}^{n} \gamma_{j} p\left(t_{j}\right)\left(\frac{\|u\|}{\sqrt{m}}\right)^{\mu} \\
\leq & \frac{\beta}{2}\|u\|^{2}-\frac{\gamma\|u\|^{\mu}}{(\sqrt{m})^{\mu}}-\sum_{j=1}^{n} \gamma_{j} p\left(t_{j}\right)\left(\frac{\|u\|}{\sqrt{m}}\right)^{\mu} .
\end{aligned}
$$

Therefore, we can choose $u_{1}$ with $\left\|u_{1}\right\| \geq T_{1}$ sufficiently large such that $\varphi\left(u_{1}\right)<0$. Then we have $\max \left\{\varphi\left(u_{0}\right), \varphi\left(u_{1}\right)\right\}<\inf \left\{\varphi(u): u \in \partial B_{T_{0}}\right\}$. And Lemma 2.7 has shown that $\varphi$ satisfies Palais-Smale condition. Hence, by Lemma 2.1 there exists a critical point $u^{*}$. Therefore, $u_{0}$ and $u^{*}$ are two critical points of $\varphi$, and they are classical solutions of (1.1).

Theorem 3.2. Suppose $\left(\mathrm{H}_{1}\right),\left(\mathrm{H}_{2}\right)$ and $a_{1}>-m / p(1)$ hold. Moreover, $f(t, u)$ and $I_{j}(u)$ are odd about $u$. Then the BVP (1.1) has infinitely many classical solutions. 
Proof. We will apply Lemma 2.3 to show this result. Firstly, $\varphi$ is even, since $f(t, u)$ and $I_{j}(u)$ are odd about $u$. Moreover, by Lemma 2.7, we know that $\varphi \in C^{1}(H, \mathbb{R}), \varphi(0)=0$, and $\varphi$ satisfies the Palais-Smale condition. In the same way as in Theorem 3.1, one can easily verify that the conditions (a) and (b) of Lemma 2.3 are satisfied. According to Lemma $2.3, \varphi$ possesses infinitely many critical points, i.e. the BVP (1.1) has infinitely many classical solutions.

\section{Example}

To illustrate how our main results can be used in practice we present the following example:

Example 4.1. Let $b_{1}>0, b(t) \in C[0,1]$ with $b(t)>0$. Consider the following boundary value problem

$$
\left\{\begin{array}{l}
\left(e^{-t} u^{\prime}(t)\right)^{\prime}+e^{-t} u(t)+b(t) u^{3}=0 \quad \text { for all } t \in[0,1], t \neq 1, \\
-\Delta u^{\prime}\left(t_{1}\right)=b_{1} u^{3} \\
u(0)=0, \quad e^{-1} u(1)+u^{\prime}(1)=0 .
\end{array}\right.
$$

CONCLUSION. BVP (4.1) has infinitely many classical solutions.

Proof. Let $p(t)=q(t)=e^{-t}, p(t)-q(t)=0, f(t, u)=b(t) u^{3}, I(u)=b_{1} u^{3}$, $\mu=4, \delta=(1 / 4) \min \{b(t): t \in[0,1]\}, \gamma=(1 / 4) \max \{b(t): t \in[0,1]\}$. Then by simple computation, the conditions in Theorem 3.2 are satisfied. Hence, by Theorem $3.2,(4.1)$ has infinitely many classical solutions. The proof is completed. $\square$

\section{REFERENCES}

[1] M. Benchohra, J. Henderson and S.K. Ntouyas, Impulsive Differential Equations and Inclusions, vol. 2, Hindawi Publishing Corporation, New York, 2006.

[2] Y.-K. ChAng AND J.J. NiETo, Existence of solutions for impulsive neutral integrodifferential inclusions with nonlocal initial conditions via fractional operators, Numer. Funct. Anal. Optim. 30 (2009), 227-244.

[3] V. Lakshmikantham, D.D. Bainov and P.S. Simeonov, Theory of Impulsive Differential Equations, World Scientific, Singapore, 1989.

[4] X. LIU AND A.R. WILlms, Impulsive controllability of linear dynamical systems with applications to maneuvers of spacecraft, Math. Probl. Eng. Aeorosp. Sci. 2 (1996), $277-$ 299.

[5] Z. LuO AND J.J. Nieto, New results for the periodic boundary value problem for impulsive integro-differential equations, Nonlinear Anal. 70 (2009), 2248-2260.

[6] J. Mawhin and M. Willem, Critical Point Theory and Hamiltonian Systems, SpringerVerlag, Berlin, 1989.

[7] X. Meng ET AL., Dynamic analysis of Michaelis-Menten chemostat-type competition models with time delay and pulse in a polluted environment, J. Math. Chem. 47 (2010), $123-144$.

[8] J.J. Nieto AND D. O'Regan, Variational approach to impulsive differential equations, Nonlinear Anal. 10 (2009), 680-690. 
[9] J.J. Nieto And R. Rodriguez-Lopez, Boundary value problems for a class of impulsive functional equations, Comput. Math. Appl. 55 (2008), 2715-2731.

[10] S. PAsquero, Ideality criterion for unilateral constraints in time-dependent impulsive mechanics, J. Math. Phys. 46 (2005), 20 pages.

[11] P.H. Rabinowitz, Amer. Math. Soc., Providence, RI 65 (1986).

[12] A.M. Samollenko And N.A. Perestyuk, Impulsive Differential Equations, World Scientific, Singapore, 1995.

[13] Y. Tian And W.G. GE, Variational methods to Sturm-Liouville boundary value problem for impulsive differential equations, Nonlinear Anal. 72 (2010), 277-287.

[14] W.B. Wang, J.H. Shen and J.J. Nieto, Permanence periodic solution of predator prey system with Holling type functional response and impulses, Discrete Dyn. Nat. Soc. 2007, 15 pages, Article ID 81756.

[15] J. Xiao And J.J. Nieto, Variational approach to some damped Dirichlet nonlinear impulsive differential equations, J. Franklin Inst. 348 (2011), 369-377.

[16] J. YAN, A. ZhAO AND J.J. NiETo, Existence and global attractivity of positive periodic solution of periodic single-species impulsive Lotka-Volterra systems, Math. Comput. Modelling 40 (2004), 509-518.

[17] S.T. Zavalishchin And A.N. Sesekin, Dynamic Impulse Systems. Theory and applications, Mathematics and its Applications, vol. 394, Kluwer Academic Publishers Group, Dordrecht, 1997.

[18] E. ZeIdLer, Nonlinear Functional Analysis and its Applications, III. Variational Methods and Optimization, Springer-Verlag, Berlin, 1985.

[19] G. Zeng, F. WAng And J.J. Nieto, Complexity of a delayed predator-prey model with impulsive harvest and Holling-type II functional response, Adv. Complex Syst. 11 (2008), 77-97.

[20] H. Zhang, L.S. Chen And J.J. Nieto, A delayed epidemic model with stage structure and pulses for management strategy, Nonlinear Anal. Real World Appl. 9 (2008), 17141726.

[21] Z. Zhang AND R. YUAN, An application of variational methods to Dirichlet boundary value problem with impulses, Nonlinear Anal. 11 (2010), 155-162.

JiNG XIAO

Department of Information Engineering

Guangdong Medical College

Dongguan

Guangdong 523808, P.R. CHINA

E-mail address: xjazyh@163.com

JuAn J. NiETO

Departamento de Análisis Matemático

Facultad de Matemáticas

Universidad de Santiago de Compostela

15782 Santiago de Compostela, SPAIN

E-mail address: juanjose.nieto.roig@usc.es

Zhiguo Luo Department of Mathematics

Hunan Normal University

Changsha

Hunan 410081, P.R. CHINA

E-mail address: luozg@hunnu.edu.cn

TMNA : Volume $43-2014-\mathrm{N}^{\circ} 2$ 